\title{
The effects of meprobamate upon the aversive threshold of rats
}

\author{
VINCENT P. HOUSER \\ Psychotropic Drug Laboratory, Veterans Administration Hospital, Perry Point, Maryland 21902
}

\begin{abstract}
An attempt was made to assay the analgesic potency of orally administered meprobamate $(50,100 \mathrm{mg})$ in the rat, using the spatial preference technique. This drug was able to raise the aversive threshold when administered in a dose of $100 \mathrm{mg}$. This same dose, however, also significantly reduced the number of motor responses made during threshold testing. These results were interpreted to suggest that meprobamate produces its effects on the aversive threshold indirectly by inhibiting the execution of the escape response.
\end{abstract}

In order to measure the analgesic properties of various agents, our laboratory has modified a technique first introduced by Campbell (1956) which uses a spatial preference cage to determine the aversive threshold of rats to grid shock. This technique allows animals to escape various shock intensities by simply crossing from one side of the cage to the other. Previous reports from this laboratory have indicated that this technique is an extremely reliable and sensitive measure of drug-induced analgesia produced by a wide variety of analgesic agents known to be clinically active in man. For example, the technique is sensitive to a number of weak analgesics (i.e., sodium salicylate, indomethacin [Houser \& Paré, 1973 b]), narcotic antagonist analgesics (i.e., pentozocine, cyclazocine [Houser \& Paré, 1973a]), as well as the classic narcotic analgesics (i.e., morphine [Houser \& Paré, 1972]), codeine, and meperidine hydrochloride [Houser \& Paré, 1973a]). The procedure also appears to be selective in that sedative doses of sodium pentobarbital which have been reported to be nonanalgesic in man (Goodman \& Gilman, 1970) are also inactive in the spatial preference technique (Houser \& Paré, 1973a).

Since this technique appears to be a sensitive and selective measure of drug-induced analgesia, we have used it to evaluate the analgesic properties of a number of tranquilizing agents. Recent evidence has suggested that both minor (i.e., chlordiazepoxide) and major (i.e., chlorpromazine) tranquilizers can elevate the aversive threshold to electric shock in the rat (Houser \& Paré, 1973b; Houser \& Van Hart, 1974). These elevations, however, are correlated with consistent decreases in motor activity. This fact may suggest that these agents could elevate the aversive threshold indirectly by blocking the execution of the escape response by means

The author would like to express his appreciation to Mr. Randall J. Cash for his technical assistance and to Mrs. Dale A. Van Hart for her help in the preparation of this manuscript. Thanks are also due to Mr. Luther R. Gilliam, Chief of the Medical Illustration Service, VA Hospital, Perry Point, Maryland, for performing the photographic work. of the sedative properties of these two drugs. To further explore the activity of tranquilizing agents, the present report explored the effects of various doses of meprobamate upon the aversive threshold of rats.

\section{METHOD}

\section{Subjects and Apparatus}

Six male Sprague-Dawley-derived rats obtained from Hilltop Lab Animals, Inc., Scottdale, Pennsylvania, were used in the present study. They weighed $220-258 \mathrm{~g}$ at the beginning of the experiment.

The test chamber and apparatus have been described in detail elsewhere (Houser \& Paré, 1972). Briefly, the chamber consisted of a rectangular Plexiglas shuttlebox which was pivoted in the middle, allowing the box to tilt from side to side as the animal crossed from one end to the other. This tilting movement activated a light action Acro lever switch, located at one end of the cage, which controlled the presentation of shock. The stainless steel rods which formed the floor of the cage could be electrified by various intensities of shock. The shock stimulus was provided by a dc generator which produced a $60-\mathrm{Hz}$-square wave output (Reus, Houser, \& Paré, 1971). Standard electromechanical scheduling and recording equipment was used to automatically present the various shock intensities and to record the amount of time in seconds spent on the shock side of the cage and number of crossing responses made during each intensity.

\section{Procedure}

Each animal was subjected to a 50 -min experimental session, the same time each day, 6 days a week. An experimental session consists of five $10-\mathrm{min}$ periods in which five separate current intensities (i.e., 30, 60, 90,120,150 microA) were presented in an ascending order. The shock was presented continuously on one side of the cage for $5 \mathrm{~min}$ and then switched to the other side for the remaining $5 \mathrm{~min}$ of each current intensity. The animal could escape the shock side of the cage by merely crossing to the opposite or nonshock portion of the tilt cage. The shock was automatically switched from one side to the other every $5 \mathrm{~min}$ to insure that each animal sampled all shock intensities even if it failed to make a crossing response during the 10 -min period that each intensity was presented.

The dependent measure consisted of the amount of time in seconds spent on the shock of the cage for each shock intensity. The aversive threshold was calculated daily for each animal by determining the intensity of shock which an animal avoided $75 \%$ of the time. At subthreshold intensities, the animal, by chance, would spend $50 \%$ of the time on the shock side of the cage. 


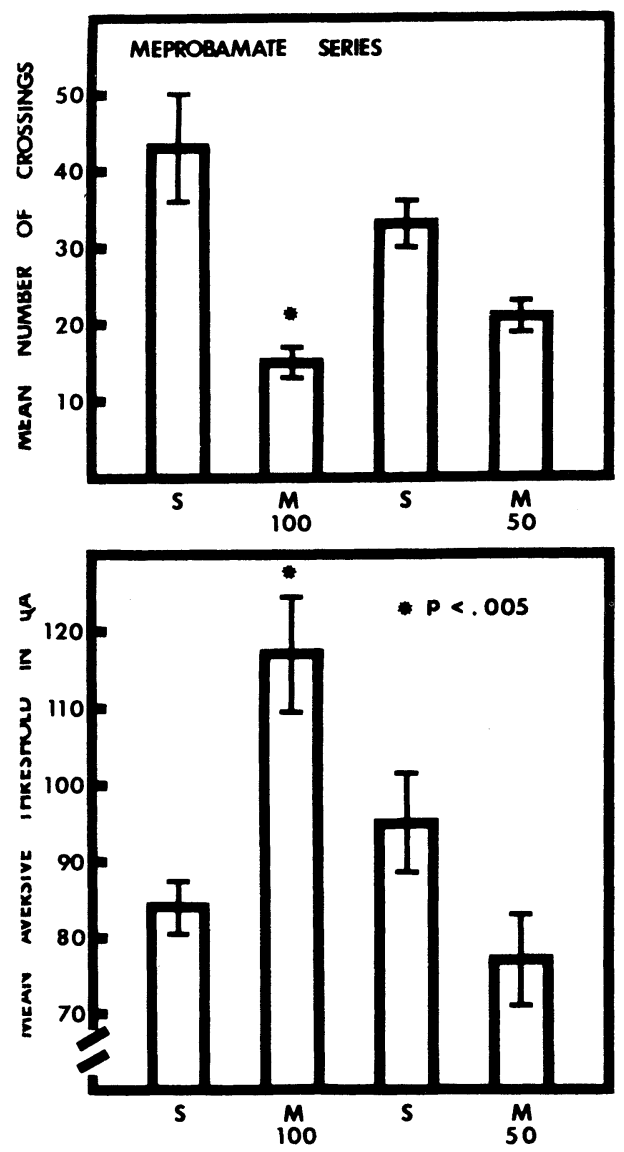

Figure 1. Mean aversive threshold and mean number of crossings made with corresponding standard error of the means for six rats subjected to various doses of meprobamate. All doses are in milligrams. Each bar represents the mean of three consecutive saline (S) or drug sessions.

Since time spent on the shock side diminished as the shock intensity increased, the $75 \%$ threshold criterion required a simple interpolation process. If animals spent more than $25 \%$ of the available time on the shock side at the highest intensity (i.e., 150 micro A), as was the case under some drug conditions, an aversive threshold could not be interpolated since no higher levels were presented. In these cases, a threshold value of 150 microA was arbitrarily assigned.

After 20 sessions, all animals demonstrated stable threshold values. The drug was given in two separate doses in consecutive weekly series. Saline was administered for the first 3 days of each weekly series, followed by 3 days of a particular drug dosage. Animals were not tested on the 7 th day of these weekly series.

Meprobamate (Equanil suspension-Wyeth) was administered orally in two separate doses $(50$ and $100 \mathrm{mg})$. Since Equanil suspension was supplied in only one concentration (i.e., $200 \mathrm{mg}$ per $5 \mathrm{cc}$ ), the dosages had to be administered by altering the volume of the solution given to the animals. The $50-\mathrm{mg}$ dose was delivered in a 1.25 -cc volume, while the $100-\mathrm{mg}$ dose was given in a volume of $2.5 \mathrm{cc} 50 \mathrm{~min}$ before threshold testing. On control days, an equivalent volume of $.9 \%$ saline was administered.

\section{RESULTS AND DISCUSSION}

Figure 1 presents the mean aversive thresholds and mean number of crossings made for the six animals subjected to various doses of meprobamate. Probability levels are given for comparisons (i.e., F tests) made between consecutive saline and drug sessions. As the data in Figure 1 indicate, meprobamate was able to significantly elevate the aversive threshold in a dose-dependent manner according to a two-factor within analysis of variance. The elevation in the threshold noted under $100 \mathrm{mg}$ of meprobamate, however, was correlated with significant reductions in the number of crossings emitted by the animals. These decrements in motor activity were quite severe. This fact leads to the conclusion that this tranquilizing agent produces a similar response profile to that seen with chlorpromazine (Houser \& Van Hart, 1974) and chlordiazepoxide (Houser \& Paré, 1973b) in the spatial preference technique. It would thus appear that meprobamate may produce elevations in the aversive threshold to grid shock indirectly by blocking the execution of the escape response by means of its sedative effects on motor activity.

\section{REFERENCES}

Campbell, B. A. The reinforcement difference limen (RDL) function for shock reduction. Journal of Experimental Psychology, 1956, 52, 268-272.

Goodman, L., \& Gilman, A. The Pharmacological Basis of Therapeutics. New York: MacMillan Company, 1970. P. 1194.

Houser, 'V. P., \& PARÉ, W. P. A method for determining the aversive threshold in the rat using repeated measures: Tests with morphine sulfate. Behavior Research Methods and Instrumentation, 1972, 4, 135-137.

Houser, V. P.. \& PARÉ, W. P. Measurement of analgesia using a spatial preference test in the rat. Physiology and Behavior, 1973, 10, 535-538. (a)

Houser, V. P., \& PARÉ, W. P. Analgesic potency of sodium salicylate, indomethacin, and chlordiazepoxide as measured by the spatial preference technique. Psychopharmacologia (Berl.), 1973, 32, 121-131. (b)

Houser, V. P., \& VAN HART, D. A. The effect of chlorpromazine and imipramine upon the aversive threshold of rats. Physiological Psychology, 1974, 2, 333-336.

Reus, J. F., Houser, V. P., \& Paré, W. P. An electronic constant current shock generator for low current levels. Physiology and Behavior, 1971, 7, 635-637.

(Received for publication June 26, 1975.) 